\title{
Improved Bit Rate Control for Real-Time MPEG Watermarking
}

\author{
Sugiri Pranata \\ Nanyang Technological University, School of Electrical \& Electronic Engineering (EEE), Nanyang Avenue, \\ Singapore 639798 \\ Email: sugiri@pmail.ntu.edu.sg

\section{Viktor Wahadaniah} \\ Nanyang Technological University, School of Electrical \& Electronic Engineering (EEE), Nanyang Avenue, \\ Singapore 639798 \\ Email:viktorw@pmail.ntu.edu.sg
}

\section{Yong Liang Guan}

Nanyang Technological University, School of Electrical \& Electronic Engineering (EEE), Nanyang Avenue, Singapore 639798

Email:eylguan@ntu.edu.sg

\section{Hock Chuan Chua}

Nanyang Technological University, School of Electrical \& Electronic Engineering (EEE), Nanyang Avenue, Singapore 639798

Email: ehchua@ntu.edu.sg

Received 2 April 2003; Revised 2 October 2003

\begin{abstract}
The alteration of compressed video bitstream due to embedding of digital watermark tends to produce unpredictable video bit rate variations which may in turn lead to video playback buffer overflow/underflow or transmission bandwidth violation problems. This paper presents a novel bit rate control technique for real-time MPEG watermarking applications. In our experiments, spread spectrum watermarks are embedded in the quantized DCT domain without requantization and motion reestimation to achieve fast watermarking. The proposed bit rate control scheme evaluates the combined bit lengths of a set of multiple watermarked VLC codewords, and successively replaces watermarked VLC codewords having the largest increase in bit length with their corresponding unmarked VLC codewords until a target bit length is achieved. The proposed method offers flexibility and scalability, which are neglected by similar works reported in the literature. Experimental results show that the proposed bit rate control scheme is effective in meeting the bit rate targets and capable of improving the watermark detection robustness for different video contents compressed at different bit rates.
\end{abstract}

Keywords and phrases: bit rate control, digital watermarking, real-time watermarking, quantized DCT watermarking.

\section{INTRODUCTION}

The rapid advancement in digital multimedia technology has brought many benefits, such as ease of creating, editing, storing, preserving, transmitting, and processing of multimedia contents. However, the same advancements have also given rise to increasing concerns over the protection of intellectual property (IP) rights. Digital watermarking has thus been proposed to help address such concerns. It is a technique to embed hidden information, called the watermark, irremovably and imperceptibly into some audio, image, or video contents, called the host, by subtly modifying their perceptual data. The embedded watermark may carry information about the origin, status, and/or destination of the host data [1], hence it can be used to facilitate the proof of ownership, provide data integrity checks, or trace the pirates. With the help of digital watermarking, it is hoped that content providers or owners will have better means to uphold their IP rights.

In many real-time multimedia applications, such as video-on-demand and video streaming, the video data is stored and transmitted in some digital compression for- 


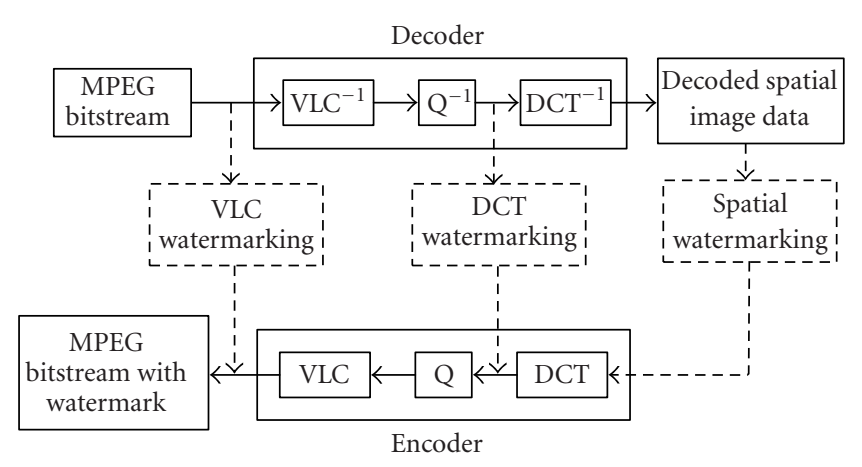

FIgURE 1: MPEG watermarking domains.

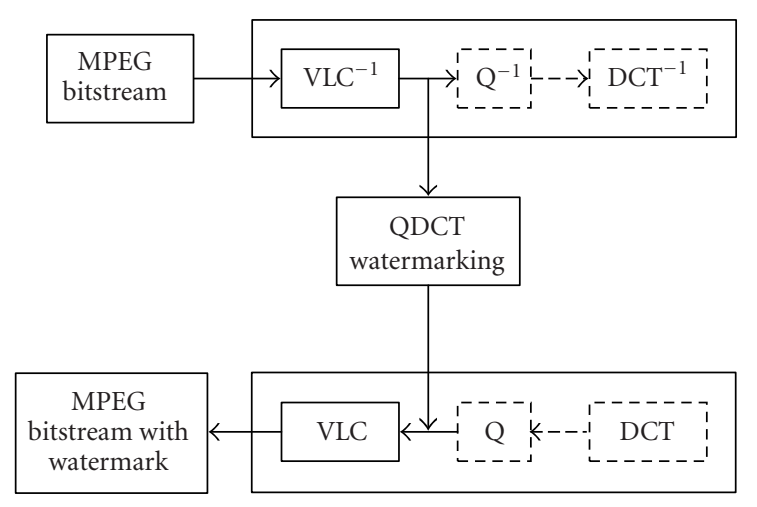

FIGURE 2: QDCT-domain watermarking for MPEG video. mats, such as MPEG. For the purpose of pirate tracing, the host video bitstream may be embedded with recipientspecific or transaction-specific information, and the resultant watermarked video will be MPEG-encoded before being transmitted to the recipients. For such applications, typically the watermark embedding process will be required to be performed in real time, and the resultant watermarked bitstream will be required to have a compression bit rate that is either the same as, or within certain permissible limits of, that before embedding. In such scenarios, therefore, fast/efficient watermarking techniques with good bit rate control provisions for MPEG video bitstream are desirable.

Existing video watermarking systems embed the watermark in various domains within the MPEG encoding process, as depicted in Figure 1.

In a spatial watermarking system $[2,3,4]$, the host video bitstream must undergo the complete MPEG decompression process which consists of inverse variable length coding $\left(\mathrm{VLC}^{-1}\right)$, inverse quantization $\left(\mathrm{Q}^{-1}\right)$, inverse discrete cosine transform (DCT) $\left(\mathrm{DCT}^{-1}\right)$, and motion compensation (not shown in Figure 1). A spatial watermarking module then embeds watermark data into the frame pixel data. The resultant watermarked data then undergoes complete MPEG recompression with motion estimation to generate a watermarked MPEG bitstream. Alternatively, a more efficient watermarking system can embed watermark in the DCT (frequency) domain $[5,6,7,8,9]$. This requires only partial MPEG decompression of the host data, and the watermark is embedded into the DCT coefficients. The resultant watermarked DCT data is then recompressed back into a watermarked MPEG bitstream with or without motion estimation.

The above two watermarking systems require MPEG decompression and recompression steps which are computationally intensive. If motion estimation is performed in the recompression process, the computational load is further increased. Consequently, these watermarking systems may not be suitable for real-time watermarking applications. To avoid the MPEG decompression and recompression steps, watermarking in the bitstream domain has been proposed $[10,11,12,13]$. In this approach, watermark data is embedded at the bitstream level by means of mapping between selected variable length coding (VLC) codewords that do not differ significantly in bit length. However, this approach is not very secure as it can be attacked by rewatermarking, and the set of VLC codewords used can be deduced fairly easily by a third party.

In this paper, we propose to perform watermarking in the quantized DCT (QDCT) domain, as shown in Figure 2. The proposed approach does not require operations, such as quantization, dequantization, DCT, or inverse DCT, to be performed. It only requires VLC and inverse VLC operations which can be efficiently implemented by table lookup. Moreover, as the embedded watermark data is not subjected to requantization, its detection robustness is more predictable.

In the design of compressed video watermarking system, especially for streaming video applications, the compression bit rate after watermark embedding is another important system parameter to consider. In the streaming video player, the video data undergoes playback through buffering. Being constrained by its capacity, the buffer fills and empties its contents at the specified bit rate within designated periods of time, so as to prevent buffer overflow or underflow. Buffer overflow occurs when the playback buffer is full, but continues to be fed with more data; buffer underflow takes place when the playback buffer runs out of data. Both situations may give rise to undesirable perceptual distortions during the video playback [14]. Watermark embedding alters the number of bits in the original video bitstream, so a good watermarking system should employ a mechanism, known as bit rate control, to control the extent of such alterations so that the problems of video playback buffer overflow or underflow can be avoided. Likewise, bit rate control can also help to prevent transmission bandwidth violations.

The concept of bit rate control for compressed video watermarking was pioneered by Hartung and Girod in [7, 15]. In their approach, each watermarked VLC codeword is 
compared with the corresponding unmarked host VLC codeword in terms of bit length. The watermarked VLC codeword is permitted only if it contains the same or a smaller number of bits compared to the host VLC codeword. Otherwise, the watermarked VLC codeword will be discarded and replaced by the host VLC codeword in the output bitstream. We denote this scheme as "Hartung 1 " in this paper. In a more elaborate scheme denoted as "Hartung 2" in this paper, if a watermarked VLC codeword contains fewer bits than the unmarked one, the unused bit length is stored as a bit budget for use in a future watermarked VLC codeword. Clearly, these bit rate control techniques may remove a sizeable proportion of the embedded watermark and hence reduce its detection robustness. Also, the resultant watermarked bitstream tends to have lower bit rate than the host bitstream. Both reductions are host-dependent and difficult to predict or control. In [16], Alattar et al. proposed a bit rate control scheme for watermarking low bit rate MPEG4 video. The proposed scheme eliminates (sets to zero) selected nonzero DCT coefficients until the target bit rate is met. As both the host and watermark data are discarded in this scheme, the authors admit that in some instances, instead of just reducing the amount of the embedded watermark the resultant video quality is compromised.

In this paper, we propose a novel bit rate control scheme to alleviate some of the shortcomings explained above. Details of both the proposed QDCT watermarking and bit rate control mechanisms will be elaborated in the next section.

\section{PROPOSED MPEG WATERMARKING SCHEME WITH BIT RATE CONTROL}

We propose watermark embedding in the QDCT domain without performing requantization and motion reestimation. This provides an efficient compressed video watermarking system for use in real-time multimedia applications, such as video-on-demand. It is assumed that the host and watermarked videos are compressed using the MPEG format. To generate watermarked video with low perceptual distortion while achieving high watermark detection robustness, spread spectrum watermarking which allows the watermark designers to control the strength and location of the watermark is employed. To control the video bit rate after watermarking, bit rate control is applied on the watermarked bitstream. A block diagram of the proposed system is shown in Figure 3.

Two common approaches for watermark embedding are considered, namely, additive and multiplicative embedding. The strength of the former lies in its simplicity, while the latter is favored for its adaptiveness to the host video data [17]. These spread spectrum watermark embedding operations can be mathematically expressed as

$$
\hat{v}_{i}= \begin{cases}v_{i}+\alpha \cdot \beta \cdot p_{i} \cdot b_{i}, & \text { for additive embedding, } \\ v_{i}+\alpha \cdot \beta \cdot p_{i} \cdot b_{i} \cdot\left|v_{i}\right|, & \text { for multiplicative embedding, }\end{cases}
$$

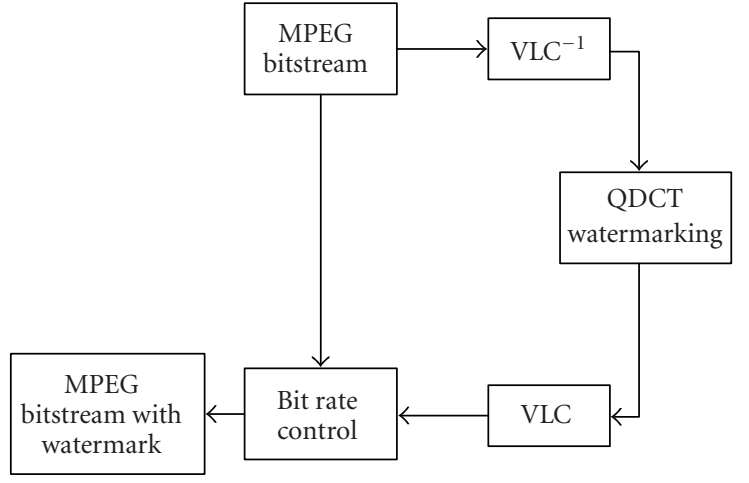

FIGURE 3: QDCT-domain watermarking with bit rate control.

where $\hat{v}_{i}$ is the watermarked data, $v_{i}$ are nonzero QDCT coefficients of the host video data at selected midfrequency locations, $\alpha$ is a user-controlled watermark amplitude scaling factor, $\beta$ is a scaling factor determined by suitable compresseddomain human visual system (HVS) modeling $[6,18,19], p_{i}$ is a pseudonoise $(\mathrm{PN})$ sequence used for spreading and despreading the watermark information bits, and $b_{i}$ is the watermark information bit of \pm 1 values. Each watermark information bit is embedded in $s$ locations, where $s$ is called the spreading factor or chip rate [7].

Blind retrieval of the embedded watermark data can be achieved by despreading the watermarked video data using the well-known correlation detector with or without prefiltering. The correlator output, hereinafter denoted by $Z$, can be compared with a suitable threshold to produce the estimated watermark information bit. Assuming that the spreading factor $s$ is large enough such that the statistical distribution of $Z$ is approximately Gaussian with mean $\mu_{Z}$ and variance $\sigma_{Z}^{2}$, and the detection threshold is set to 0 (i.e., the watermark information bit is estimated by taking the sign of $Z$ ), the bit error probability or bit error rate (BER) of the blind watermark retrieval process can be shown to be

$$
\mathrm{BER}=Q\left(\sqrt{\frac{\mu_{Z}^{2}}{\sigma_{Z}^{2}}}\right)=Q(\sqrt{\mathrm{SNR}})
$$

where $Q(x)=(1 / \sqrt{2 \pi}) \int_{x}^{\infty} e^{-t^{2} / 2} d t$ and SNR $=\mu_{Z}^{2} / \sigma_{Z}^{2}$ is the signal-to-noise ratio (SNR) of the correlator output. Generally, when this correlator output SNR value is high, the corresponding BER will be low and hence the watermark will be robust.

Next, we will describe our proposed bit rate control scheme. Note that although it is discussed in conjunction with QDCT-domain watermark embedding in this paper, it can also be used with other watermarking schemes including spatial-domain and DCT-domain watermarking.

Essentially, our proposed bit rate control scheme compares the total number of bits (bit length) of two buffers: 


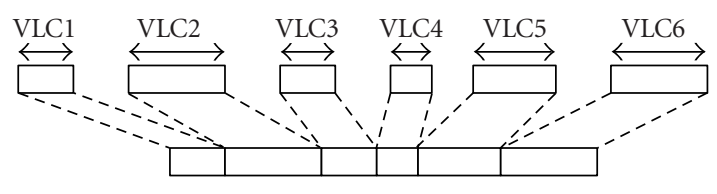

FIgURe 4: A host buffer containing six successive host VLC codewords, before watermark embedding.

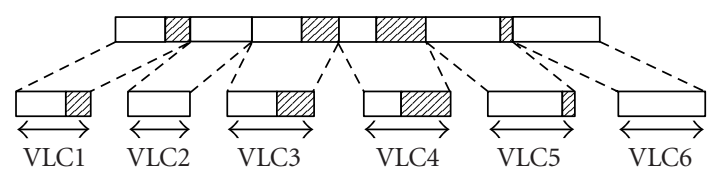

FIGURE 5: Output buffer containing six successive VLC codewords in the watermarked bitstream, corresponding to those shown in Figure 4, but after watermark embedding.

one (called the output buffer) contains VLC codewords in a segment of the watermarked bitstream; the other (called the host buffer) contains the corresponding VLC codewords in the host bitstream. If the bit length of the output buffer is greater than that of the host buffer by a user-specified threshold $T$, watermarked VLC codewords in the output buffer with the first few largest increase in number of bits will be successively restored to their unmarked counterparts (i.e., the corresponding host VLC codewords) until the total bit length of the output buffer is no larger than that of the host buffer by $T$. Once this is achieved, the contents of the resultant output buffer will form the final watermarked bitstream with controlled bit rate. This process of checking and restoring "excessively long" watermarked VLC codewords is repeated for the entire watermarked bitstream.

For illustration, a host buffer containing a segment of six successive host VLC codewords is shown in Figure 4.

After watermark embedding, the six corresponding VLC codewords in the watermarked bitstream are placed in the output buffer as shown in Figure 5. Some of these output buffer codewords are longer in bit length than their host counterparts. For example, the watermarked VLC codewords VLC1, VLC3, VLC4, and VLC5 have increase in bit length indicated by the shaded regions in Figure 5, whereas VLC2 and VLC6 have a reduction and no change in bit length, respectively.

Assuming that the total bit length of the output buffer in Figure 5 exceeds that of the host buffer in Figure 4 by more than the user-specified threshold $T$, then the watermarked VLC codewords in the output buffer will be modified as follows. First, the watermarked VLC codewords are sorted by the amount of increase in bit length in a descending order, as shown in Figure 6.

Then, the VLC codeword with the largest increase in bit length is replaced with its original host VLC codeword. In the example shown in Figure 6, VLC4 is therefore replaced with its corresponding host codeword. The total bit length

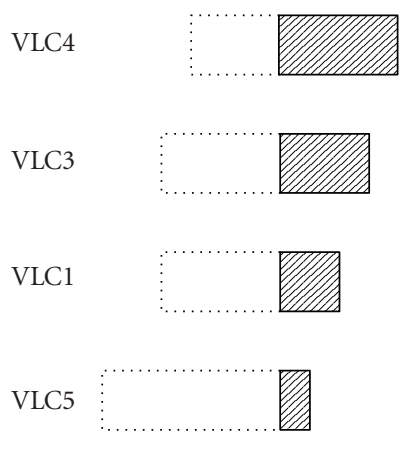

FIGURE 6: Watermarked VLC codewords descendingly sorted by increase in bit length.

of the resultant output buffer is then reexamined. If it still exceeds the host buffer length by more than $T$, then the next VLC codeword in the ordered list, VLC3, is restored. This process is continued until the length of the "shortened" output buffer falls within the specified limit, then the output buffer contents are sent as the output watermarked bitstream.

In the bit rate control mechanism described above, the number of VLC codewords in the host/output buffers is user-specified. It can be a slice, a macroblock, or any other convenient values. This parameter can be used to control the amount of watermark discarded by the bit rate control scheme, hence it has a direct impact on the detection robustness and visual quality of the final watermarked bitstream. If the host/output buffer is chosen to contain only one VLC codeword, then the earlier proposed scheme reduces to Hartung's schemes.

Another parameter of the proposed bit rate control scheme, the threshold $T$, can be used to control the target bit rate to be achieved. For example, $T=0$ will largely result in the watermarked bitstream having the same bit rate as the host bitstream, while $T>0$ will permit the watermarked bitstream to have higher bit rate than the host bitstream.

The major difference between our proposed bit rate control scheme and Hartung's schemes is that our scheme compares the total bit length of a set of VLC codewords instead of an individual VLC codeword. As we expect some VLC codewords after watermark embedding to be longer, while others to be shorter, than before embedding, their combined bit length is likely to show relatively small or no increase compared to that before embedding. Hence, our proposed scheme preserves more watermarked VLC codewords than Hartung's. This will obviously translate into a gain in robustness in the blind watermark retrieval process for our proposed scheme. In cases where watermarked VLC codewords need to be discarded due to excessive increase in bit length, our proposed strategy of restoring watermarked VLC codewords with the largest increase in bit length helps to ensure that the target bit rate is reached quickly with minimal amount of watermark discarded. Furthermore, our proposed 
scheme is also scalable as it allows the watermarked bitstream to have controlled increase in bit rates from the host bitstream. This is not possible in Hartung's schemes. Therefore, our proposed bit rate control scheme provides more flexibility in the design of MPEG watermarking system with variable bit rate constraints or requirements. Our proposed bit rate control does take a bit more processing time to accumulate and compare the bit lengths of the host and watermarked VLC codewords. However, this is not significant compared to the processing required by the entire watermark embedding operation. Finally, it should also be fairly obvious that since no watermarked VLC codeword is set to zero in our proposed scheme, it does not suffer from similar visual quality degradation as that encountered in Alattar's scheme [16].

\section{EXPERIMENTAL RESULTS}

In this section, the performance of the proposed bit rate control scheme is investigated and analysed via computer experiments. The watermarking source codes are developed from the MPEG-2 video codec source code provided in the MPEG Software Simulation Group website (http://www.mpeg.org/ MPEG/MSSG). Three MPEG-1 video sequences encoded with main profile are used for testing; they are, namely, susie.mpg, flower.mpg, and table-tennis.mpg. The three sequences contain 450 frames with 38 I-frames. Each frame consists of $352 \times 240$ pixels (CIF resolution). Although MPEG-1 format is used here for illustration, the results and conclusions obtained are expected to be applicable to MPEG2 and MPEG-4 videos, too.

Several watermark amplitudes $(\alpha=1,2$, and 3 for additive embedding; $50 \%, 100 \%$, and $150 \%$ for multiplicative embedding) are used to generate different watermarked MPEG bitstream. To ensure low visual distortion, the watermarks are embedded selectively into the nonzero QDCT coefficients of the luminance blocks from the 16th to the 43rd zigzag-scan frequency locations. For each video, four watermarking scenarios are studied.

(1) Watermarking with no bit rate control technique (hereinafter denoted as "None").

(2) Watermarking with our proposed bit rate control technique (hereinafter denoted as "Proposed") with host/output buffer size set to 1 macroblock, and buffer threshold $T$ set to zero, that is, to maintain the video bit rate to be the same before and after watermarking.

(3) Watermarking with "Hartung 1" bit rate control technique (described earlier in Section 1).

(4) Watermarking with "Hartung 2" bit rate control technique (described earlier in Section 1).

In each case, random watermark information bits are embedded at a spreading rate of one information bit per Iframe, and retrieved by correlation without prefiltering. This trial is repeated 1000 times and the resultant correlation outputs are collated to calculate its mean $\mu_{Z}$ and variance $\sigma_{Z}^{2}$. The robustness of the retrieved watermark is measured in terms of the correlator output SNR $=\mu_{Z}^{2} / \sigma_{Z}^{2}$. This robustness measure is adopted instead of BER because the corresponding BER values are very low and hence tedious to obtain experimentally. Nonetheless, the expected BER values can be estimated from the correlator output SNR values by using (2). The quality of the watermarked video is measured in terms of peak-signal-to-noise ratio (PSNR) averaged over all frames [20], taken with reference to the MPEG host video of a specified compression bit rate.

First of all, we investigate the effect of different bit rate control techniques on the resultant watermarked video bit rate under variation of watermark amplitude, as shown in Figure 7. The host video bit rate is set to $1.5 \mathrm{Mbps}$. It is shown in Figure 7 that the bit rate of the watermarked video without bit rate control is always larger than the host bit rate. This may lead to the problem of video playback buffer overflow or transmission bandwidth violation. Furthermore, the bit rate increments are different for different videos, watermark embedding approaches (additive or multiplicative embedding), and watermark amplitude scaling factors. This variability may present uncertainties and difficulties to the watermark designers.

In contrast, such tendency does not occur to the watermarked videos generated using Hartung's or our proposed bit rate control technique. However, the watermarked videos obtained using Hartung's techniques are observed to have consistently lower bit rate than the host video. This is not surprising because in "Hartung 1," only watermarked VLC codewords with equal or smaller bit length, compared to their original VLC codewords, are allowed to be embedded. "Hartung 2" produces slightly higher bit rate than "Hartung 1 " because some unused bit length is allowed to be stored. Nonetheless, the algorithm checks VLC codewords consecutively and such extra bit length may not be always utilized. As a result, the watermarked bitstream generated under Hartung's methods tends to contain shorter VLC codewords than the host bitstream. This may lead to video playback buffer underflow or result in low hiding capacity. On the contrary, watermarked videos generated based on our proposed technique successfully maintain their original bit rate at $1.5 \mathrm{Mbps}$.

Next, Figure 8 plots the correlator output SNR and the PSNR of the watermarked video generated using different watermark amplitude values. The results show that under the same watermark amplitude, different bit rate control techniques give rise to different degrees of watermark detection robustness (quantified by the correlator output SNR) and visual quality (quantified by PSNR). Specifically, watermarking without bit rate control leads to the highest correlator output SNR and correspondingly lowest PSNR; watermarking with Hartung's bit rate control schemes achieves exactly the opposite; while watermarking with our proposed scheme achieves somewhere in between. Such performance differences can be easily explained by the fact that no watermark is lost if no bit rate control is applied, while "Hartung 1" results in the highest amount of embedded watermark being discarded. More watermark embedded in the video leads to 


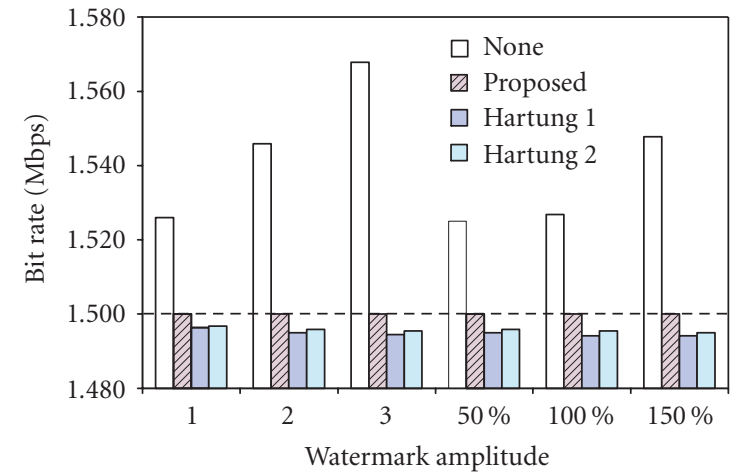

(a)

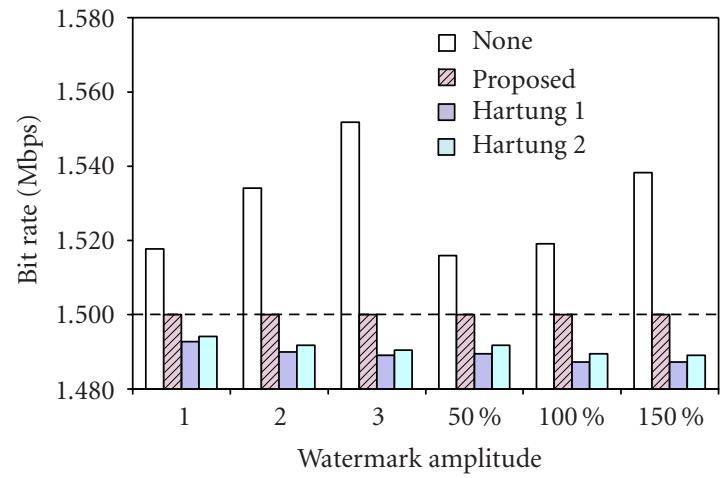

(b)

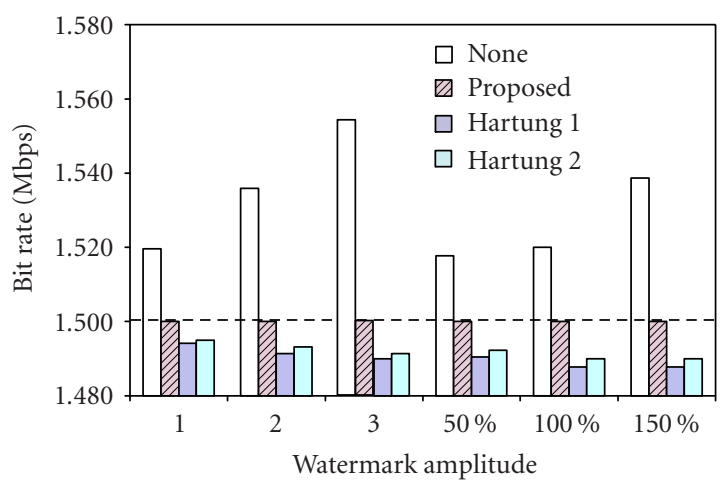

(c)

FIGURE 7: Bit rate of watermarked video generated using different bit rate control schemes under watermark amplitude variation (additive embedding $\alpha=1,2,3$; multiplicative embedding $\alpha=50 \%, 100 \%, 150 \%$ ). Host videos are (a) susie.mpg, (b) flower.mpg, and (c) tabletennis.mpg (all compressed at $1.5 \mathrm{Mbps}$ ).

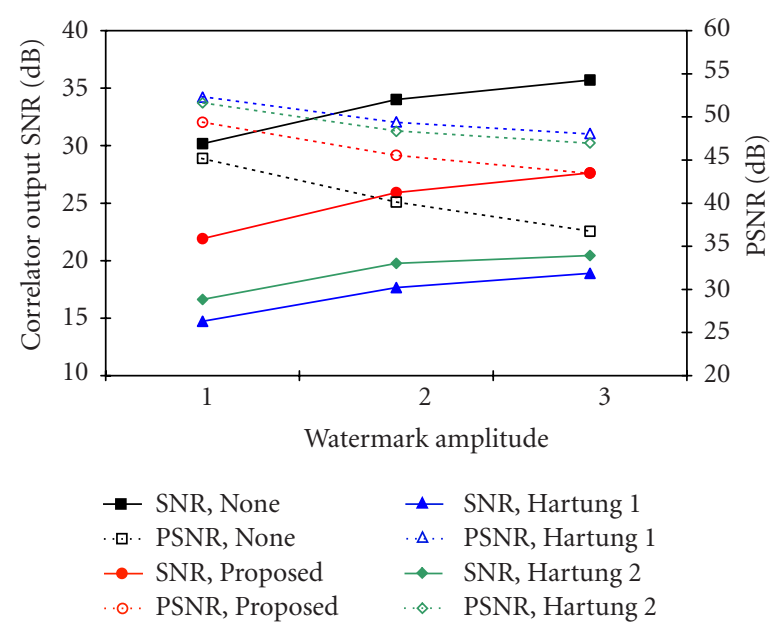

(a)

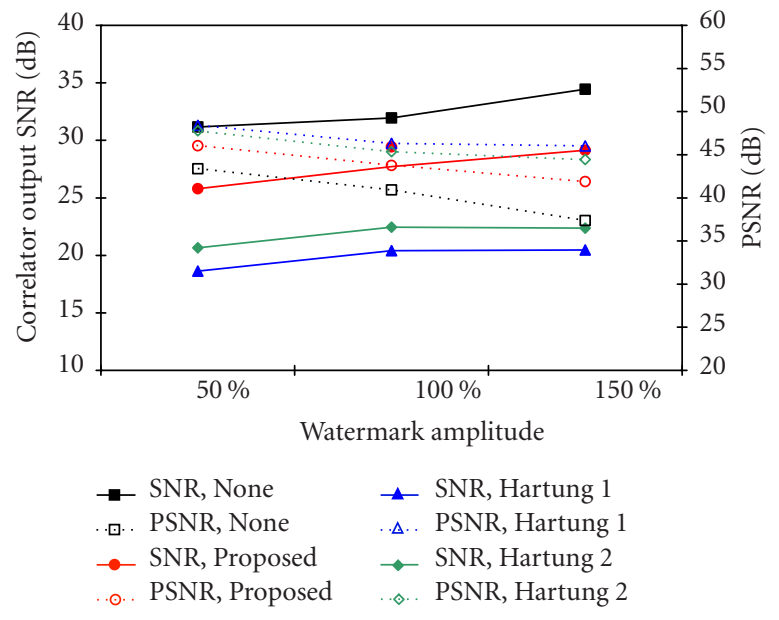

(b)

FIGURE 8: Correlator output SNR and PSNR versus watermark amplitude of different bit rate control techniques using (a) additive embedding and (b) multiplicative embedding. Host video susie.mpg is compressed at $1.5 \mathrm{Mbps}$. 


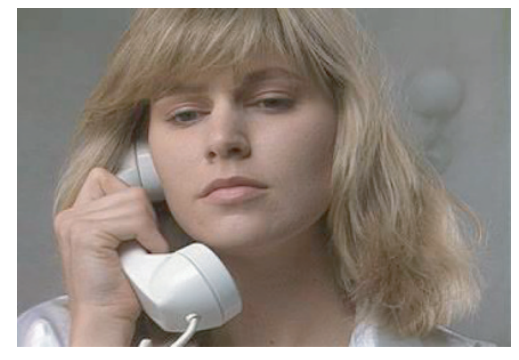

(a)

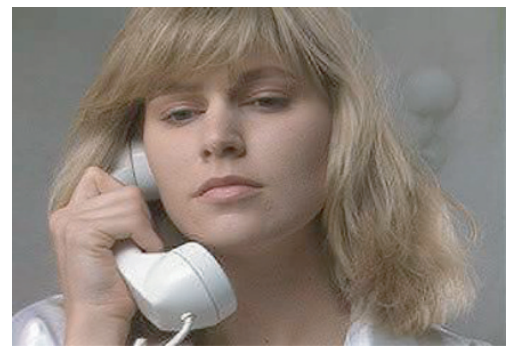

(b)

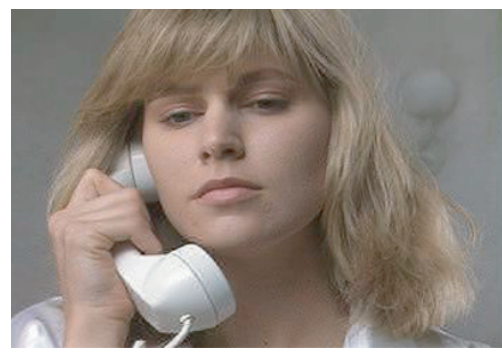

(c)

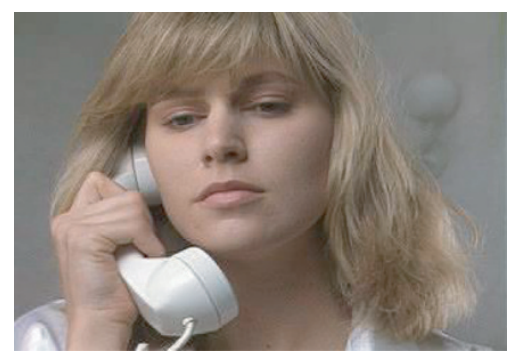

(d)

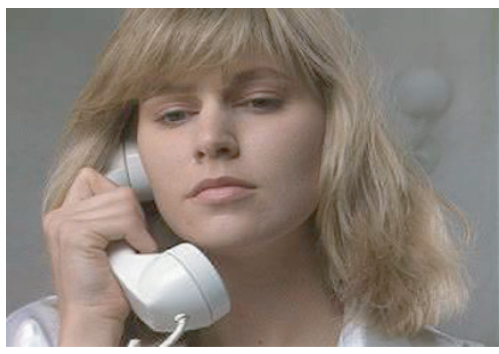

(e)

FIGURE 9: Frames of susie.mpg video (host video compressed at 1.5 Mbps) as (a) unmarked, (b) watermarked with "None," (c) watermarked with "Proposed," (d) watermarked with "Hartung 1," and (e) watermarked with "Hartung 2."

better detection quality (higher correlator output SNR) but more visual distortion (lower PSNR). For illustration, video frames watermarked using different bit rate control schemes with $\alpha$ of $100 \%$ are shown in Figure 9. Their visual qualities are found to be generally acceptable with corresponding PSNR values exceeding $40 \mathrm{~dB}$, compared to the unmarked host frame.

To better quantify the relative performance of the various bit rate control schemes, Figure 10 plots the correlator output SNR against the PSNR of watermarked video bitstreams generated using different bit rate control schemes with additive or multiplicative embedding. It shows that for the same visual quality after watermark embedding, our proposed bit rate control technique is able to improve the watermark detection robustness vis-à-vis Hartung's schemes. Equivalently, at the same degree of detection robustness, our proposed bit rate control technique is capable of producing much less perceptual distortion than Hartung's, as indicated by the higher PSNR value. This is true for all the test videos, as well as for both additive and multiplicative watermark embedding approaches.

For the susie.mpg and table-tennis.mpg videos, Figures 10a and 10c show that the improvement in correlator output SNR achieved by our scheme over Hartung's can be as large as $5 \mathrm{~dB}$. For the flower.mpg video, Figure $10 \mathrm{~b}$ even indicates that, over a range of PSNR from 31 to $35 \mathrm{~dB}$ approximately, our proposed bit rate control scheme can produce higher correlator output SNR than if no bit rate control is used. This is despite the fact that watermarking without bit rate control produces the highest video bit rate and does not suffer loss in watermark. This peculiar phenomenon can be explained by the histogram of the correlator output shown in Figure 11. It shows that while the mean $\mu_{Z}$ of the correlator output $(Z)$ increases progressively from "Hartung 1 " to "Hartung 2," then to our proposed scheme, and finally to the "no bit rate control" scheme, the variance $\sigma_{Z}^{2}$ of $Z$ for the "no bit rate control" scheme is also the largest. Therefore, its correlator output SNR value, which is given by the ratio $\mu_{Z}^{2} / \sigma_{Z}^{2}$, turns out to be lower than the correlator output SNR value of our proposed scheme (which is clearly seen to have the smallest $\sigma_{Z}^{2}$ in Figure 11).

Finally, the performance differences between our proposed bit rate control scheme and Hartung's schemes under different MPEG compression bit rates $(0.5 \mathrm{Mbps}$ and $1.5 \mathrm{Mbps}$ ) are shown in Figures 12 and 13 for additive and multiplicative watermarking respectively. It shows that all bit rate control schemes experience lower correlator output SNR values for the $0.5 \mathrm{Mbps}$ video. This is because there is generally less hiding capacity in video with low compression rates. Nonetheless, our proposed bit rate control technique is observed to achieve fairly consistent improvement over Hartung's under different MPEG bit rates. This demonstrates the versatility of our proposed bit rate control scheme. 


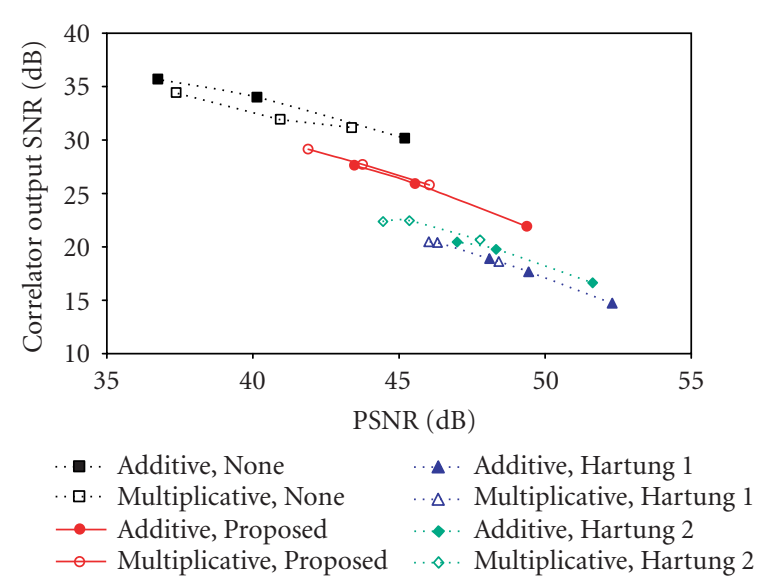

(a)

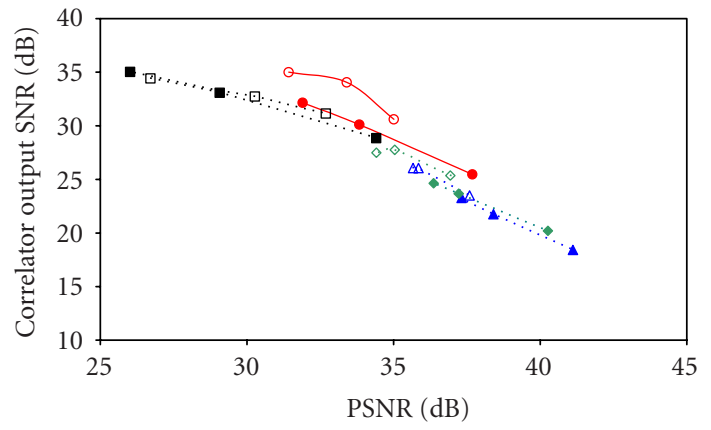

-. Additive, None

๑.. Multiplicative, None

$\ldots$ Additive, Proposed

- Multiplicative, Proposed $\star \cdots$ Additive, Hartung 1
$\Delta \cdots$ Multiplicative, Hartung 1
$\star \cdots$ Additive, Hartung 2
$\diamond \cdots$ Multiplicative, Hartung 2

(b)

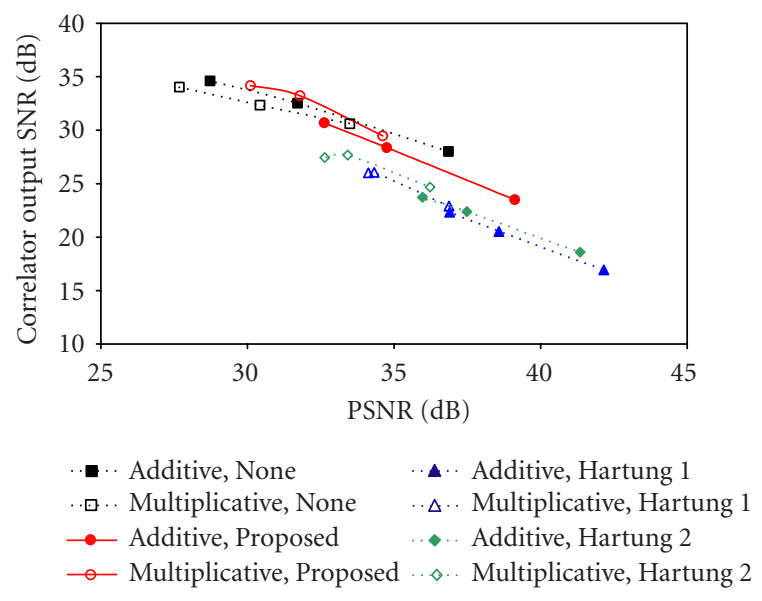

(c)

FIGURE 10: Correlator output SNR versus PSNR of watermarked bitstreams generated using different bit rate control schemes for (a) susie.mpg, (b) flower.mpg, and (c) table-tennis.mpg (all compressed at $1.5 \mathrm{Mbps}$ before watermark embedding).

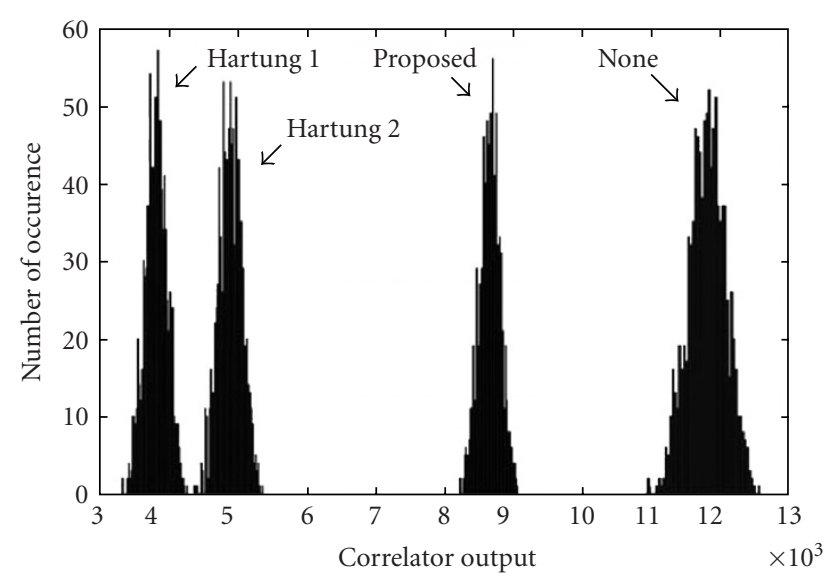

FIGURE 11: Histograms of correlator output for flower.mpg with multiplicative watermark $(\alpha=100 \%)$ and different bit rate control schemes.

\section{CONCLUSIONS}

In this paper, we present a novel bit rate control technique for real-time MPEG video watermarking to better control the bit rate of watermarked video bitstream. The proposed bit rate control scheme evaluates the combined bit length of multiple watermarked VLC codewords, and successively replaces watermarked VLC codewords having the largest increase in bit length with their corresponding unmarked VLC codewords until a target bit length is achieved. The proposed bit rate control technique offers greater scalability than similar works reported in the literature. Experimental results also show that it is effective in meeting bit rate targets and capable of improving the detection reliability/robustness of the embedded watermark for different video contents and different MPEG compression bit rates. Although the proposed bit rate control technique is discussed in conjunction with QDCT-domain watermark embedding in this paper, it can also be used with any other watermarking schemes including spatial-domain and DCT-domain watermarking. 


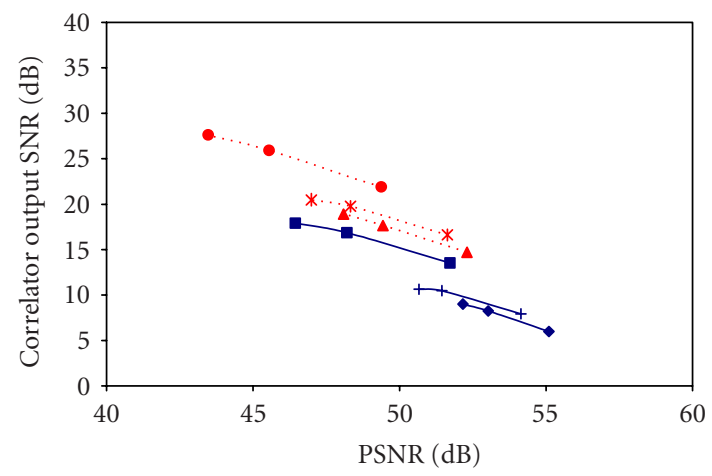

-. Proposed, $1.5 \mathrm{Mbps} \rightarrow$ Proposed, $0.5 \mathrm{Mbps}$

.4. Hartung 1, 1.5 Mbps $\rightarrow$ Hartung 1, 0.5 Mbps

*- Hartung 2, $1.5 \mathrm{Mbps} \rightarrow$ Hartung 2, $0.5 \mathrm{Mbps}$

(a)

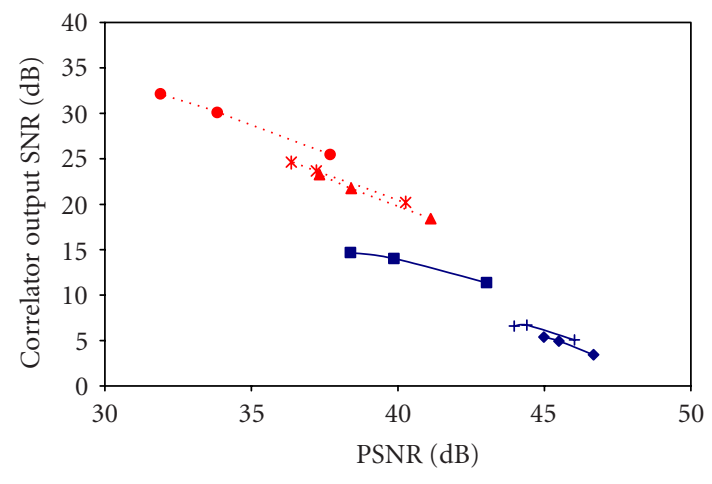

- Proposed, $1.5 \mathrm{Mbps} \rightarrow$ Proposed, $0.5 \mathrm{Mbps}$

... Hartung 1, 1.5 Mbps $\rightarrow$ Hartung 1, 0.5 Mbps

* Hartung 2, 1.5 Mbps † Hartung 2, $0.5 \mathrm{Mbps}$

(b)

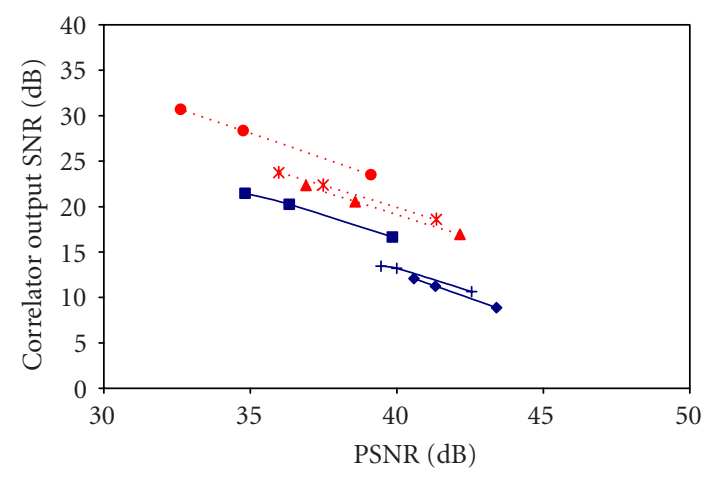

- Proposed, $1.5 \mathrm{Mbps} \rightarrow$ Proposed, $0.5 \mathrm{Mbps}$

4. Hartung 1, 1.5 Mbps $\rightarrow$ Hartung 1, 0.5 Mbps

* Hartung 2, 1.5 Mbps T Hartung 2, 0.5 Mbps

(c)

FIgURE 12: Correlator output SNR versus PSNR for (a) susie.mpg, (b) flower.mpg, and (c) table-tennis.mpg with different MPEG compression bit rates before watermark embedding and different bit rate control techniques after additive watermark embedding.

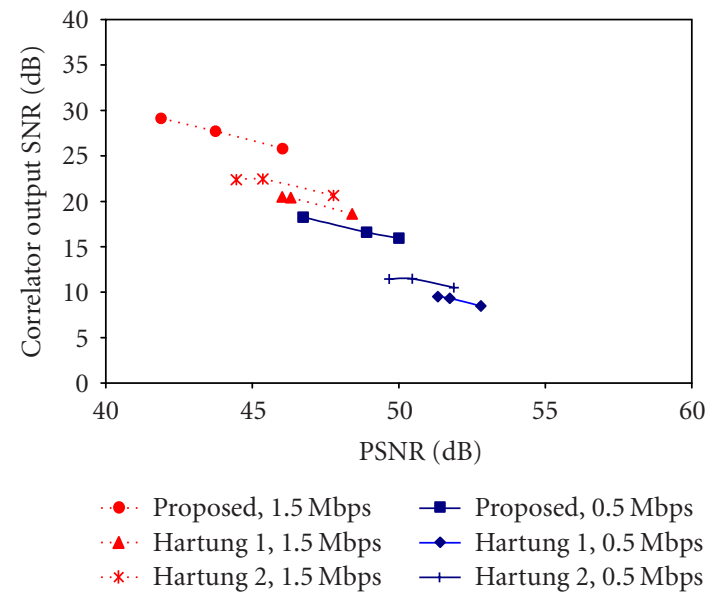

(a)

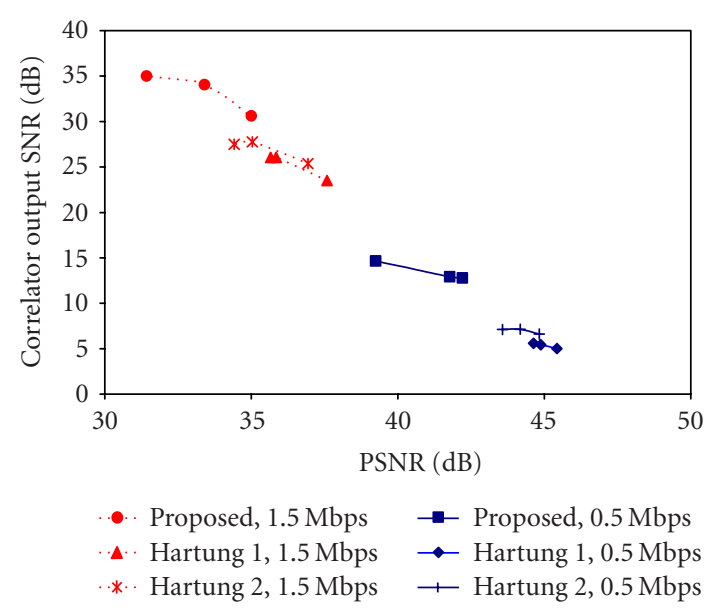

(b)

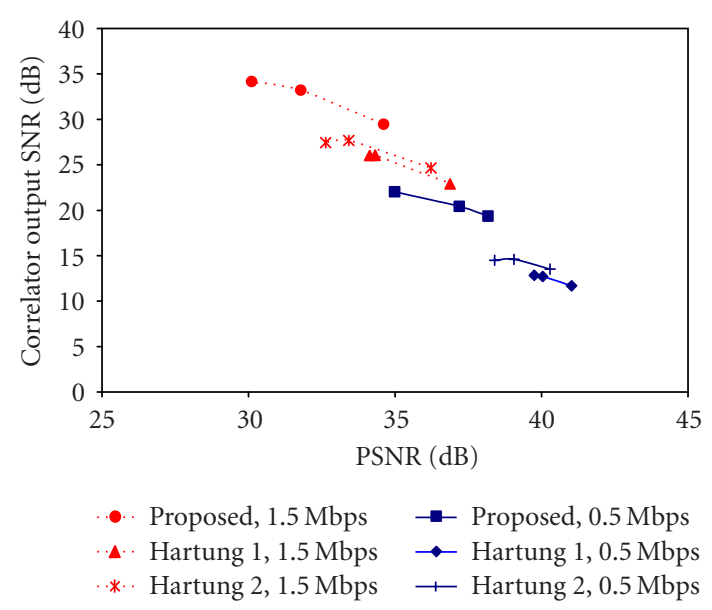

(c)

FIGURE 13: Correlator output SNR versus PSNR for (a) susie.mpg, (b) flower.mpg, and (c) table-tennis.mpg with different MPEG compression bit rates before watermark embedding, and different bit rate control techniques after multiplicative watermark embedding. 


\section{REFERENCES}

[1] F. Hartung and M. Kutter, "Multimedia watermarking techniques," Proceedings of the IEEE, vol. 87, no. 7, pp. 1079-1107, 1999.

[2] M. George, J.-Y. Chouinard, and N. Georganas, "Digital watermarking of images and video using direct sequence spread spectrum techniques," in Proc. IEEE Canadian Conference on Electrical and Computer Engineering, vol. 1, pp. 116-121, Edmonton, Alberta, Canada, May 1999.

[3] T. Kalker, G. Depovere, J. Haitsma, and M. J. Maes, "Video watermarking system for broadcast monitoring," in Security and Watermarking of Multimedia contents, vol. 3657 of Proceedings of SPIE, pp. 103-112, San Jose, Calif, USA, January 1999.

[4] B. G. Mobasseri, "A spatial digital video watermark that survives MPEG", in Proc. IEEE International Conference on Information Technology: Coding and Computing, pp. 68-73, Las Vegas, Nev, USA, March 2000.

[5] M. Barni, F. Bartolini, V. Cappellini, and A. Piva, "A DCTdomain system for robust image watermarking," Signal Processing, vol. 66, no. 3, pp. 357-372, 1998.

[6] T.-Y. Chung, M.-S. Hong, Y.-N. Oh, D.-H. Shin, and S.H. Park, "Digital watermarking for copyright protection of MPEG2 compressed video," IEEE Transactions on Consumer Electronics, vol. 44, no. 3, pp. 895-901, 1998.

[7] F. Hartung and B. Girod, "Watermarking of uncompressed and compressed video," Signal Processing, vol. 66, no. 3, pp. 283-301, 1998.

[8] C.-T. Hsu and J.-L. Wu, "DCT-based watermarking for video," IEEE Transactions on Consumer Electronics, vol. 44, no. 1, pp. 206-216, 1998.

[9] T. L. Wu and S. F. Wu, "Selective encryption and watermarking of MPEG video," in Proc. International Conference on Image Science, Systems, and Technology (CISST '97), Las Vegas, Nev, USA, June-July 1997.

[10] A. Hanjalic, G. C. Langelaar, P. M. B. Van Roosmalen, et al., Image and Video Databases: Restoration, Watermarking and Retrieval, Part II, chapters 7-9, Elsevier Science, New York, NY, USA, 2000.

[11] G. C. Langelaar, "Feasibility of security concept in hardware", SMASH, Tech. Rep. SMS-TUD-633-1, Philips Research Labs, The Netherlands, August 1996.

[12] G. C. Langelaar, R. L. Lagendijk, and J. Biemond, "Real-time labeling methods for MPEG compressed video," in Proc. 18th Symposium on Information Theory in the Benelux, Veldhoven, The Netherlands, May 1997.

[13] G. C. Langelaar, R. L. Lagendijk, and J. Biemond, "Real-time labeling of MPEG-2 compressed video," Journal of Visual Communication and Image Representation, vol. 9, no. 4, pp. 256-270, 1998.

[14] ISO/IEC 11172-2:1993 (E), Information technology-Coding of moving pictures and associated audio for digital media at up to about 1.5 Mbit/s, Part 2: Video, Switzerland.

[15] F. Hartung and B. Girod, "Digital watermarking of raw and compressed video," in Digital Compression Technologies and Systems for Video Communications, vol. 2952 of Proceedings of SPIE, pp. 205-213, Berlin, Germany, October 1996.

[16] A. M. Alattar, M. U. Celik, and E. T. Lin, "Evaluation of watermarking low-bit-rate MPEG-4 bit streams," in Security and Watermarking of Multimedia Contents V, vol. 5020 of Proceedings of SPIE, pp. 440-451, Santa Clara, Calif, USA, January 2003.

[17] M. Barni, C. I. Podilchuk, F. Bartolini, and E. J. Delp, "Watermark embedding: hiding a signal within a cover image," IEEE Communications Magazine, vol. 39, no. 8, pp. 102-108, 2001.
[18] C. Busch, W. Funk, and S. Wolthusen, "Digital watermarking: from concepts to real-time video applications," IEEE Computer Graphics and Applications, vol. 19, no. 1, pp. 25-35, 1999.

[19] M. Lee and G. Reynolds, "Edge detection using DCT coefficients in MPEG video," Tech. Rep. 01/28, CSIRO Mathematical and Information Sciences, Melbourne, Australia, 2001.

[20] M. Kutter and F. A. Petitcolas, "Fair benchmark for image watermarking systems," in Security and Watermarking of Multimedia Contents, vol. 3657 of Proceedings of SPIE, pp. 226-239, San Jose, Calif, USA, January 1999.

Sugiri Pranata received his B.Eng. and M.Eng. degrees from the School of EEE, Nanyang Technological University, Singapore. His research interests include video signal processing and watermarking.

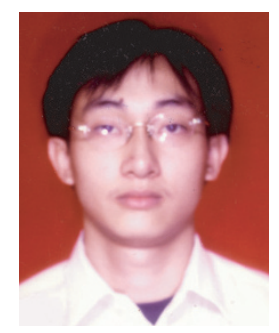

Viktor Wahadaniah received his B.Eng. and M.Eng. degrees from the School of EEE, Nanyang Technological University, Singapore. His research interests include digital watermarking and video signal processing.

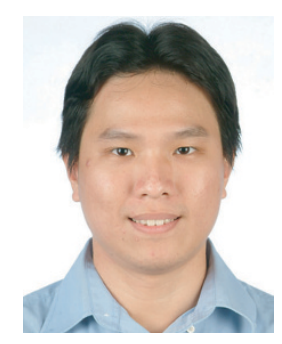

Yong Liang Guan received his B.Eng. and Ph.D. degrees from the National University of Singapore and Imperial College of Science, Technology and Medicine (University of London), respectively. He is currently an Assistant Professor with the School of EEE, Nanyang Technological University (NTU), Singapore. He is also the Program Director for the Wireless Network Research Group in the Positioning and Wireless Technology

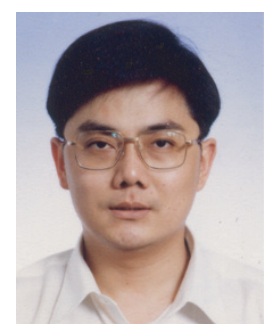
Center (PWTC), and the Deputy Director of the Center for Information Security of NTU. His research interests include digital multimedia watermarking, multicarrier modulation, turbo and spacetime coding/decoding, and communication channel modeling.

Hock Chuan Chua is currently an Associate Professor in the School of Electrical and Electronic Engineering of Nanyang Technological University, Singapore. His research interests include video signal processing, watermarking, and Internet applications.

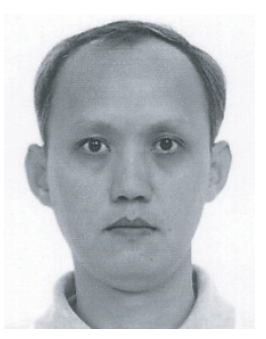

